\title{
De novo formation of a central nervous system cavernous malformation: implications for predicting risk of hemorrhage
}

\section{Case report and review of the literature}

\author{
Paul W. Detwiler, M.S., M.D., Randall W. Porter, M.D., Joseph M. Zabramski, M.D., and Robert \\ F. Spetzler, M.D.
}

Division of Neurological Surgery, Barrow Neurological Institute, Mercy Healthcare Arizona, Phoenix, Arizona

The authors present a documented sporadic de novo cavernous malformation of the central nervous system in a patient undergoing follow-up magnetic resonance imaging after resection of an acoustic neuroma. The authors believe that this is the first report of a de novo cavernous malformation in a patient without a familial history of this disease or a history of treatment with cranial radiation. The occurrence of de novo lesions invalidates the common assumption that cavernous malformations are congenital lesions. The use of this assumption to calculate bleeding risks retrospectively in patients with cavernous malformations is likely to underestimate the risk of symptomatic hemorrhage significantly.

Consequently, the de novo formation of cavernous malformations may be more common than appreciated and may explain the higher bleeding rates reported in prospective compared with retrospective studies of these lesions.

Key Words * cavernous malformation * de novo formation * hemorrhage * vascular malformation

Cavernous malformations are low-flow, hemorrhagic vascular lesions that affect 0.4 to $0.5 \%$ of the population.[3,23] In general, it has been assumed that they are congenital lesions.[3,11,13,22] The occurrence and development of de novo cavernous malformations challenge this assumption.[6-8,15-18,27-29] The de novo formation of cavernous malformations is well recognized in patients affected by the familial form of the disease and has been reported at a rate of 0.4 lesions per patient per year.[29] A less common but established group of patients at risk for genesis of de novo cavernous malformations are those undergoing radiotherapy. New lesions have been described after both whole-brain and stereotactic irradiation.[7,16-18,28]

Treatment decisions regarding the management of cavernous malformations require an understanding of their natural history. These lesions enlarge and produce symptoms as a result of repeated episodes of hemorrhage. Investigators studying the natural history of cavernous malformations have calculated annual hemorrhage rates by means of both prospective and retrospective data. The retrospective calculation of hemorrhage rates is based on the assumption that all cavernous malformations are present 
from birth.[3,11,13,22] In contrast, prospective studies track patients in whom lesions have been radiographically documented over a given period of time for clinical or radiographic evidence of hemorrhage.[1,23,29] Such prospective studies are consistently associated with a higher rate of symptomatic hemorrhage than retrospective studies. The de novo formation of cavernous malformations offers a simple explanation for this discrepancy and suggests that bleeding rates are underestimated by retrospective studies. This report describes a patient with a sporadic de novo cavernous malformation of the central nervous system and reviews the literature on this topic.

\section{CASE REPORT}

History. In 1991, this 32-year-old woman underwent resection of a right acoustic neuroma. The tumor was discovered on magnetic resonance (MR) imaging after the patient developed acute onset of right-sided tinnitus, hearing loss, and decreased facial sensation. No other lesions were documented at that time. The tumor was resected via the retrosigmoidal approach. After surgery, MR imaging of the brain with and without gadolinium enhancement showed only postoperative changes (Fig. 1 left). In 1995, a follow-up gadolinium-enhanced MR study of the brain revealed a new multilobulated lesion in the right parietal region (Fig. 1 right). The signal intensity of the center of the lesion was high, inhomogeneous, and surrounded by a rim of low signal intensity. These findings were considered consistent with a de novo cavernous malformation. The patient denied any family history of seizures, stroke, or other symptoms consistent with intracranial vascular lesions. The risks and benefits of surgery along with the reported range of hemorrhage rates were presented to the patient, and she selected surgical removal of the lesion.
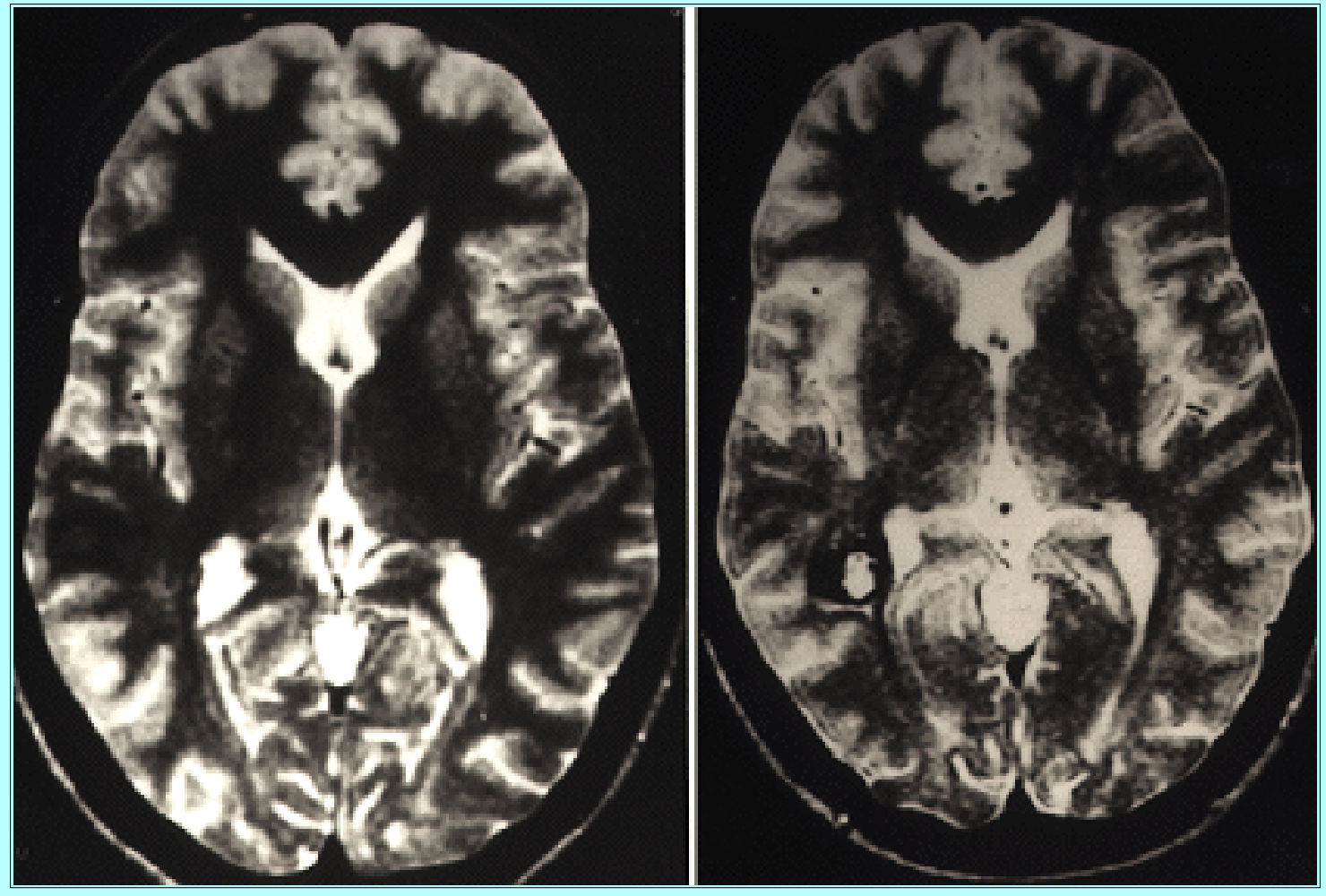

Fig. 1. Left: Axial $T_{2}$-weighted MR image of the brain obtained in 1991. There is no suggestion of a cavernous malformation in the right periatrial area. Right: $\mathrm{A} \mathrm{T}_{2}$-weighted MR image of the brain obtained in 1995. The lesion in the right periatrial area demonstrates the classic $T_{2}$-weighted appearance of a cavernous malformation: a multilobulated central area of high signal intensity with a surrounding rim of low signal intensity. 
Operation. In January 1996 the new lesion was resected via a frameless stereotactic ISG wand-guided (ISG Technologies, Inc., Mississauga, ON, Canada) right parietal craniotomy. The lesion was verified pathologically to be a cavernous malformation. No neurological deficit occurred from the resection of the cavernous malformation.

\section{DISCUSSION}

\section{Origin of Cavernous Malformations}

The origin of cavernous malformations is likely multifactorial. Cranial radiation, genetic, and viral causes all have been implicated.[6-8,16-18,27-29] Pozzati and coworkers[18] studied 18 patients with new and aggressive cerebral cavernous malformations. Of six patients harboring de novo lesions, four had previously undergone irradiation. Three had received high-dose cranial radiotherapy and one had been treated with stereotactic radiation therapy. Viral infection also may play a role in producing or triggering the formation of cavernous malformations. In immunodeficient rats the polyoma virus has been used to induce the formation of multiple intracranial cavernous malformations. $[6,8,27]$ There is also a report of the formation of a new lesion along the path used to obtain a biopsy specimen of a deep subcortical cavernous malformation.[15] The authors of this report attributed the new cavernous malformation to iatrogenic seeding from the lesion.

Genetic factors have been identified in 20 to $30 \%$ of patients with cavernous malformations. The familial, or hereditary, form of this disease appears to be most common among Hispanics but has been reported among most ethnic groups.[4,9,10,14,16,19,29] Genetic analyses of affected families have demonstrated an autosomal-dominant mode of inheritance. Linkage studies have localized the genetic mutation responsible for familial cavernous malformations to the long arm of chromosome 7.[4,9,10,12] Based on MR screening studies, penetrance approaches $100 \%$ in affected adults; however, only approximately $60 \%$ of those affected have clinically recognizable symptoms. Zabramski, et al.,[29] followed six unrelated families with documented histories of familial cavernous malformations. Serial MR imaging at 6- to 12-month intervals in 21 patients documented the formation of 17 new lesions during a mean follow up of 2.2 years. The incidence of clinically symptomatic hemorrhage in this group of patients was $6.5 \%$ per patient per year.

Other authors have speculated that cavernous malformations are part of a developmental spectrum of vascular malformations that include capillary telangiectasis, cavernous malformations, and arteriovenous malformations. In support of their pathogenic theory, they cite the reported coexistence of these lesions as well as intermediate forms.[2,14,20,22,28] Russell and Rubinstein[25] argue against classifying capillary telangiectasia as a developmental precursor to cavernous malformations. They note, "If the cavernous angioma represents a latter stage of development of the telangiectasis, the latter should be observed in greater numbers and in earlier life than the former." The association of cavernous malformations with venous malformations,[2,16,17,21,22,24,26,28] capillary telangiectasis,[16,17,20,22,25,28] and gliomas[5,28] has been well established in humans.

In this report, the 32-year-old woman who presented with a de novo cavernous malformation in the right parietal region of the brain had no history consistent with familial cavernous malformations. Her initial surgery for the acoustic neuroma 4 years earlier included both pre- and postoperative MR studies that were negative for cavernous malformation or other vascular abnormalities. It is possible that the cavernous malformation was present but missed on the initial MR images. The studies, however, were 
performed on the same high-field, 1.5-tesla imager (GE Signa Scanner; GE Medical Systems, Milwaukee, WI) using similar sequences that included gradient-echo imaging. This latter sequence is extremely sensitive to the presence of even small cavernous malformations. To the best of our knowledge, therefore, this is the first report--based on two separate MR studies--demonstrating evidence of a de novo cavernous malformation in the absence of the familial form of the disease or radiation therapy to the brain.

Pozzati, et al.,[16] may also have reported such a patient, a 36-year-old woman with recurrent seizures, who presented with a de novo cavernous malformation during pregnancy. She had no history of familial cavernous malformations or radiation. This patient had undergone a previous computerized tomography (CT) scan of the head, which was described but not shown in the publication, that was unremarkable. Follow-up MR imaging revealed a temporal cavernous malformation. Therefore, this lesion could represent a true de novo cavernous malformation, or the CT scan may not have been sensitive enough to detect the lesion. In a separate publication, Pozzati, et al.,[18] presented a cavernous malformation that was initially missed on a CT scan; it was later detected on MR imaging and then retrospectively identified on the original CT scan. Cavernous malformations are frequently missed on CT scanning. In a series of patients undergoing both CT and MR imaging, Rigamonti, et al.,[19] reported that CT scanning demonstrated only nine of 18 lesions visualized by MR imaging.

\section{Risk of Hemorrhage}

A number of factors, including age, sex, and presentation, can complicate the interpretation of prospective versus retrospective studies when trying to ascertain the true risk of hemorrhage for cavernous malformations. Retrospective calculations of hemorrhage rate assume that all lesions are present from birth and that the hemorrhage rate is relatively uniform throughout life. Using these assumptions, Curling, et al.,[3] calculated a retrospective hemorrhage rate of $0.1 \%$ per lesion per year in a group of 32 patients over 2890 patient-years. In 122 patients, Kondziolka, et al.,[13] calculated a retrospective hemorrhage rate per patient of $1.3 \%$ over 4551 patient-years.

Robinson, et al.,[23] reported the results of a prospective study of 57 patients with cavernous malformations that had been documented on MR imaging. During a mean 26-month follow up, one patient suffered a symptomatic hemorrhage, giving a bleeding rate for the series of $0.7 \%$ per lesion per year. The only hemorrhage in this group was in a pregnant woman, leading the authors to speculate that pregnancy may increase the risk of recurrent bleeding.

Aiba and colleagues[1] followed a group of 110 patients with cavernous malformations for a mean of 4.7 years and reported that the risk of hemorrhage varied with age and sex. The risk of bleeding was highest in females younger than 40 years of age ( $34 \%$ per lesion per year) compared with $22 \%$ for a group of males in the same age group. The authors did not correlate the risk of bleeding with pregnancy. In contrast, Kondziolka, et al.,[13] noted no difference in hemorrhage rates in a prospective study of 60 males and 62 females followed for a mean of 34 months, whereas Robinson, et al.,[23] found no statistical evidence to support a correlation between age and risk of hemorrhage in patients with cavernous malformations.

Several authors have reported that the risk of symptomatic hemorrhage varies significantly with the type of clinical presentation. Aiba, et al.,[1] noted that the risk of hemorrhage was greatest in the subgroup of patients with "overt" hemorrhage (defined as the acute or subacute onset of focal neurological deficits, signs of intracranial hypertension in conjunction with a fresh hemorrhage outside the cavernous 
malformation at the time of surgical extirpation, or hemorrhage seen on an initial CT scan consistent with an intralesional or perilesional hematoma that had resolved on follow-up CT scanning). During a mean follow-up period of 4.7 years, the risk of hemorrhage was $22.9 \%$ per year in 62 patients who presented with overt hemorrhage, compared with $0.39 \%$ per year in 23 who presented with seizures, and zero in 23 patients with incidental lesions. Kondziolka, et al.,[13] support these findings: in a prospective study, 122 patients with MR-documented cavernous malformations were followed for a mean of 34 months. The clinical risk of bleeding was $0.6 \%$ per year in patients with incidental lesions compared with $4.5 \%$ in patients who presented with a previous symptomatic hemorrhage.

\section{CONCLUSIONS}

Cavernous malformations are dynamic lesions that grow and produce symptoms by repeated episodes of hemorrhage. Rates of hemorrhage may vary considerably depending on the patient's age, sex, and presentation. The de novo formation of cavernous malformations also raises questions regarding the validity of the assumption that these lesions are truly congenital. These findings suggest that only prospective studies can be expected to provide accurate data regarding the risks of symptomatic hemorrhage.

\section{References}

1. Aiba T, Tanaka R, Koike T, et al: Natural history of intracranial cavernous malformations. J Neurosurg 83:56-59, 1995

2. Ciricillo SF, Dillon WP, Fink ME, et al: Progression of multiple cryptic vascular malformations associated with anomalous venous drainage. Case report. J Neurosurg 81:477-481, 1994

3. Curling OD Jr, Kelly DL Jr, Elster AD, et al: An analysis of the natural history of cavernous angiomas. J Neurosurg 75:702-708, 1991

4. Dubovsky J, Zabramski JM, Kurth J, et al: A gene responsible for cavernous malformations of the brain maps to chromosome 7q. Hum Mol Genet 4:453-458, 1995

5. Fischer EG, Sotrel A, Welch K: Cerebral hemangioma with glial neoplasia (angioglioma?). Report of two cases. J Neurosurg 56:430-434, 1982

6. Flocks JS, Weis TP, Kleinman DC, et al: Dose-response studies to polyoma virus in rats. J Natl Cancer Inst 35:259-284, 1965

7. Gaensler EHL, Dillon WP, Edwards MSB, et al: Radiation-induced telangiectasia in the brain simulates cryptic vascular malformations at MR imaging. Radiology 193:629-636, 1994

8. Gross L: Oncogenic Viruses, ed 2. Oxford: Pergamon Press, 1970, pp 651-750

9. Günel M, Awad IA, Anson J, et al: Mapping a gene causing cerebral cavernous malformation to 7q11.2-q21. Proc Natl Acad Sci USA 92:6620-6624, 1995

10. Günel M, Awad IA, Finberg K, et al: A founder mutation as a cause of cerebral cavernous malformation in Hispanic Americans. N Engl J Med 334:946-951, 1996

11. Hsu FPK, Rigamonti D, Huhn SL: Epidemiology of cavernous malformations, in Awad IA, Barrow 
DL (eds): Cavernous Malformations. Park Ridge, Ill: American Association of Neurological Surgeons, 1993, pp 13-23

12. Johnson EW, Iyer LM, Rich SS, et al: Refined localization of the cerebral cavernous malformation gene (CCM1) to a 4-cM interval of chromosome 7q contained in a well-defined YAC contig. Genome Res 5:368-380, 1995

13. Kondziolka D, Lunsford LD, Kestle JRW: The natural history of cerebral cavernous malformations. J Neurosurg 83:820-824, 1995

14. Maraire JN, Awad IA: Intracranial cavernous malformations: lesion behavior and management strategies. Neurosurgery 37:591-605, 1995

15. Ogilvy CS, Moayeri N, Golden JA: Appearance of a cavernous hemangioma in the cerebral cortex after a biopsy of a deeper lesion. Neurosurgery 33:307-309, 1993

16. Pozzati E, Acciarri N, Tognetti F, et al: Growth, subsequent bleeding, and de novo appearance of cerebral cavernous angiomas. Neurosurgery 38:662-670, 1996

17. Pozzati E, Giangaspero F, Marliani F, et al: Occult cerebrovascular malformations after irradiation. Neurosurgery 39:677-684, 1996

18. Pozzati E, Giuliani G, Nuzzo G, et al: The growth of cerebral cavernous angiomas. Neurosurgery 25:92-97, 1989

19. Rigamonti D, Hadley MN, Drayer BP, et al: Cerebral cavernous malformations. Incidence and familial occurrence. N Engl J Med 319:343-347, 1988

20. Rigamonti D, Johnson PC, Spetzler RF, et al: Cavernous malformations and capillary telangiectasia: a spectrum within a single pathological entity. Neurosurgery 28:60-64, 1991

21. Rigamonti D, Spetzler RF: The association of venous and cavernous malformations. Report of four cases and discussion of the pathophysiological, diagnostic, and therapeutic implications. Acta Neurochir 92:100-105, 1988

22. Robinson JR, Awad IA: Clinical spectrum and natural course, in Awad IA, Barrow DL (eds):

Cavernous Malformations. Park Ridge, Ill: American Association of Neurological Surgeons, 1993, pp $25-36$

23. Robinson JR, Awad IA, Little JR: Natural history of the cavernous angioma. J Neurosurg 75:709-714, 1991

24. Robinson JR Jr, Brown AP, Spetzler RF: Occult malformation with anomalous venous drainage. J Neurosurg 82:311, 1995 (Letter)

25. Russell DS, Rubinstein LJ: Pathology of Tumours of the Nervous System, ed 4. Baltimore: Williams \& Wilkins, 1977, pp 116-145

26. Sasaki O, Tanaka R, Koike T, et al: Excision of cavernous angioma with preservation of coexisting venous angioma. Case report. J Neurosurg 75:461-464, 1991

27. Vandeputte M: Antilymphocytic serum and polyoma oncogenesis in rats. Transplant Proc 
1:100-105, 1969

28. Wilson CB: Cryptic vascular malformations. Clin Neurosurg 38:49-84, 1992

29. Zabramski JM, Wascher TM, Spetzler RF, et al: The natural history of familial cavernous malformations: results of an ongoing study. J Neurosurg 80:422-432, 1994

Manuscript received March 3, 1997.

Accepted in final form May 13, 1997.

Address reprint requests to: Joseph M. Zabramski, M.D., c/o Neuroscience Publications, Barrow Neurological Institute, 350 West Thomas Road, Phoenix, Arizona 85013-4496. email: neuropub@mha.chw.edu. 